\title{
FINITELY EMBEDDED MODULES OVER GROUP RINGS
}

\author{
by P. F. SMITH \\ (Received 24th June 1976)
}

Let $R$ be a ring and $X$ a right $R$-module (all rings have identities and all modules are unitary). The intersection of all non-zero submodules of $X$ is denoted by $\mu(X)$. The module $X$ is called monolithic if and only if $\mu(X) \neq 0$ and in this case $\mu(X)$ is an essential simple submodule of $X$. (Recall that a submodule $Y$ of $X$ is essential if and only if $Y \cap A \neq 0$ for every non-zero submodule $A$ of $X$.) It is well known that a module $X$ is monolithic if and only if there is a simple right $R$-module $U$ such that $X$ is a submodule of the injective hull $E(U)$ of $U$. If $x$ is a non-zero element of an arbitrary right $R$-module $X$ then by Zorn's Lemma there is a submodule $Y_{x}$ of $X$ maximal with the property $x \notin Y_{x}$. It can easily be checked that $X / Y_{x}$ is monolithic and $\cap Y_{x}=0$, where the intersection is taken over all non-zero elements $x$ of $X$.

Vámos (15) defined a right $R$-module $X$ to be finitely embedded in case the injective hull of $X$ is a finite direct sum $E\left(S_{1}\right) \oplus E\left(S_{2}\right) \oplus \cdots \oplus E\left(S_{n}\right)$ of injective hulls of simple right $R$-modules $S_{i}, 1 \leqslant i \leqslant n$. He proved that a right $R$-module $X$ is finitely embedded if and only if its socle is a finitely generated essential submodule, and he showed that this is equivalent to $X$ having the property that for every collection of submodules $Y_{\lambda}, \lambda \in \Lambda$, of $X$ with $\cap_{\Lambda} Y_{\lambda}=0$ there exists a finite subset $\Lambda^{\prime}$ of $\Lambda$ such that $\cap_{\Lambda^{\prime}} Y_{\lambda}=0$. He also proved that a module $X$ is Artinian if and only if every homomorphic image of $X$ is finitely embedded.

A ring $R$ is a Hilbert ring if and only if $R$ is right Noetherian and the Jacobson radical of every homomorphic image of $R$ is nilpotent. A simple homomorphic image of a ring is called a capital of that ring. A field is called absolute if and only if it is an algebraic extension of a finite field. A ring $R$ is called a CHACA ring if and only if $R$ is a commutative Hilbert ring such that every capital of $R$ is an absolute field.

A group $G$ is polycyclic if and only if there is a series

$$
1=G_{0} \triangleleft G_{1} \triangleleft \cdots \triangleleft G_{n-1} \triangleleft G_{n}=G
$$

in which the factors $G_{i} / G_{i-1}, 1 \leqslant i \leqslant n$, are all cyclic. It is well known that a finitely generated nilpotent group is polycyclic. If $\mathfrak{X}$ and $\mathfrak{V}$ are group classes then a group $H$ is called an $\mathfrak{X}$-by- $\mathfrak{Y}$ group if and only if there is a normal subgroup $N$ of $H$ such that $N$ lies in $\mathfrak{X}$ and $H / N$ in $\mathfrak{Y}$.

Let $J$ be a CHACA ring, $G$ be a polycyclic-by-finite group and $X$ be a finitely generated monolithic right $J G$-module. If $U=\mu(X)$ then there is a maximal ideal $M$ of $J$ such that $U$ is finite dimensional over the field $J / M$ by a theorem of Roseblade (11, Corollary $A$ ). It follows by methods of Jategaonkar (6) that for some positive integer $k, X$ is a finitely generated module over the ring $J / M^{k}$ and in particular $X$ has 
finite length. The question arises whether monolithic $J G$-modules are Artinian and in this note we prove the following result.

Theorem A. Let $J$ be a commutative Hilbert ring and $G$ be a finitely generated nilpotent-by-finite group. Let $X$ be a finitely embedded JG-module such that the socle of $X$ is finitely generated as a $J$-module. Then $X$ is Artinian.

This result together with Roseblade's theorem mentioned above combine to give immediately:

Corollary A. Let $J$ be a CHACA ring and $G$ a finitely generated nilpotent-by-finite group. Then every finitely embedded JG-module is Artinian.

Let $K$ be any field, $G$ be a finitely generated nilpotent-by-finite group and $X$ be a finitely embedded right $K G$-module. If $K$ is absolute then $X$ is Artinian by Corollary $A$. Also it is not hard to see that if $G$ is Abelian-by-finite then $X$ is Artinian (Corollary 2.2). Otherwise the problem of whether or not $X$ is Artinian reduces to considering finitely embedded modules over capitals of $K H$ where $H$ is a finitely generated nilpotent normal subgroup of finite index in $G$. In $\$ 1$ we show that if every capital of $K H$ is Artinian then every finitely embedded $K H$-module is Artinian and in $\$ 2$ we show how this extends to finitely embedded $K G$-modules. Unfortunately, this takes us no further because of the next result.

Theorem B. Let $K$ be a field and $G$ be a finitely generated nilpotent-by-finite group such that every capital of $K G$ is Artinian. Then either $K$ is absolute or $G$ is Abelian-by-finite.

Snider (14) proved that if $K$ is a field and $G$ is a polycyclic group such that every primitive ideal of the group ring $K G$ is a maximal ideal then $K$ is absolute or $G$ is nilpotent-by-finite. We prove that if $J$ is a ring and $G$ is a group such that every primitive homomorphic image of the group ring $J G$ is Artinian then every primitive homomorphic image of $J H$ is Artinian for every normal subgroup $H$ of finite index in $G$ (Lemma 3.3). But it is well known that if $P$ is a primitive ideal of a ring $R$ and the ring $R / P$ is Artinian then $R / P$ is simple and hence $P$ is a maximal ideal. Thus combining these results with Theorem $B$ we can extend Theorem B as follows.

Theorem B'. Let $K$ be a field and $G$ be a polycyclic-by-finite group such that every primitive homomorphic image of $K G$ is Artinian. Then either $K$ is absolute or $G$ is Abelian-by-finite.

Now suppose that $J$ is a commutative Artinian ring and $G$ is a finite group. If $U$ is a simple right $J G$-module then Rosenberg and Zelinsky (13, Theorem 3) proved that the injective hull $E(U)$ has finite length, and so in particular is finitely generated. We shall prove the following result.

Theorem C. Let $J$ be a CHACA ring and $G$ be a polycyclic-by-finite group such 
that either $J$ is not Artinian or $G$ is infinite. Then there does not exist a non-zero finitely generated injective JG-module which is faithful for $J$.

I am grateful to the referee for bringing (14) to my attention. There is some overlap with (14). In particular, Theorem 3.2 is essentially the same as (14, Lemma 1).

\section{Polycentral rings}

Let $R$ be a ring. An ideal $I$ of $R$ is said to have a centralising set of generators if and only if there is a finite set of elements $c_{i}, 1 \leqslant i \leqslant n$, of $I$ and a chain

$$
O=I_{0} \subseteq I_{1} \subseteq \cdots \subseteq I_{n-1} \subseteq I_{n}=I
$$

of ideals $I_{j}$ of $R$ such that $I_{j}$ is generated by $c_{1}, c_{2}, \ldots, c_{j}$ and $c_{j}+I_{j-1}$ is a central element of the ring $R / I_{j-1}$ for all $I \leqslant j \leqslant n$. If every ideal of a ring $R$ has a centralising set of generators then we call the ring $R$ polycentral.

Let $X$ be a right $R$-module, $R$ any ring. If $S$ is a non-empty subset of $R$ then the annihilator of $S$ in $X$ will be denoted by $\operatorname{ann}_{X}(S)$; thus $\operatorname{ann}_{X}(S)=\{x \in X: x S=0\}$. If $S=\{c\}$ then we shall denote $\operatorname{ann}_{X}(S)$ simply by $\operatorname{ann}_{X}(c)$. If $T$ is a non-empty subset of $X$ then the annihilator of $T$ in $R$ is $\operatorname{ann}_{R}(T)=\{r \in R: T r=0\}$.

Lemma 1.1. Let $R$ be $a$ ring and $X$ a right $R$-module which contains an Artinian submodule $Y$. Let $c$ be a central element of $R$ such that $\operatorname{ann}_{X}(c)$ is Artinian. Then $\{x \in X: x c \in Y\}$ is an Artinian submodule of $X$.

Proof. It is clear that $A=\{x \in X: x c \in Y\}$ is a submodule of $X$. Let $A_{1} \supseteq A_{2} \supseteq$ $A_{3} \supseteq \cdots$ be a descending chain of submodules of $A$. If $B_{i}=A_{i} \cap a n_{X}(c)$ for each positive integer $i$ then $B_{1} \supseteq B_{2} \supseteq B_{3} \supseteq \cdots$ is a descending chain of submodules of ann $_{X}(c)$ and $A_{1} c \supseteq A_{2} c \supseteq A_{3} C \supseteq \cdots$ is a descending chain of submodules of $Y$. Since $a_{n}(c)$ and $Y$ are both Artinian there exists a positive integer $n$ such that $B_{n}=$ $B_{n+1}=B_{n+2}=\cdots$ and $A_{n} c=A_{n+1} c=A_{n+2} c=\cdots$. It is a simple check to show that $A_{n}=A_{n+1}=A_{n+2}=\cdots$. It follows that $A$ is Artinian.

Lemma 1.2. Let $R$ be a ring and $X$ a finitely embedded right $R$-module which is not Artinian. Then there exists an Artinian submodule $Y$ of $X$ such that $X / Y$ is not finitely embedded but $X / A$ is finitely embedded for every proper submodule $A$ of $Y$.

Proof. Let $\mathscr{S}$ be the collection of submodules $C$ of $X$ such that $X / C$ is not finitely embedded. Since $X$ is not Artinian it follows by (15, Proposition $\left.2^{*}\right)$ that $\mathscr{S}$ is non-empty. Let $\left\{S_{\lambda}: \lambda \in \Lambda\right\}$ be a chain of submodules in $\mathscr{S}$ and $S=\cap_{\Lambda} S_{\lambda}$. If $S$ does not lie in $\mathscr{S}$ then $X / S$ is finitely embedded and hence $n_{\Lambda}\left(S_{\lambda} / S\right)=0$ implies that $S_{\lambda} / S=0$ for some $\lambda$ in $\Lambda$ by $\left(15\right.$, Proposition $\left.1^{*}\right)$, a contradiction. It follows that $S$ belongs to $\mathscr{S}$. By Zorn's Lemma $\mathscr{S}$ has a minimal member $Y$. If $A$ is a proper submodule of $Y$ then $X / A$ is finitely embedded by the choice of $Y$. By (15, Proposition $3^{*}$ ) it follows that $Y / A$ is finitely embedded for every proper submodule $A$ of $Y$ and hence by (15, Proposition $\left.2^{*}\right) Y$ is Artinian. 
Corollary 1.3. Let $R$ be $a$ ring and $c$ be a central element of $R$. Let $X$ be $a$ $c$-torsion right $R$-module such that $\operatorname{ann}_{X}(c)$ is Artinian. Then $X$ is Artinian.

Proof. For every non-zero element $x$ of $X$ there exists a positive integer $k$ such that $x c^{k}=0, x c^{k-1} \neq 0$, and hence $x c^{k-1} \in \operatorname{ann}_{X}(c)$. Thus ann $n_{X}(c)$ is an essential submodule of $X$. Since $a n_{X}(c)$ is Artinian it follows that $X$ is finitely embedded by (15, Lemma 1). If $X$ is not Artinian then there exists an Artinian submodule $Y$ of $X$ such that $X / Y$ is not finitely embedded (Lemma 1.2). By Lemma 1.1 ann $_{X / Y}(c)$ is Artinian and thus $X / Y$ is finitely embedded by the first part of the proof. This contradiction gives the result.

Lemma 1.4. Let $R$ be a ring and $I$ an ideal with a centralising set of generators. Let $X$ be an I-torsion right $R$-module such that $\operatorname{ann}_{X}(I)$ is Artinian. Then $X$ is Artinian.

Proof. Let $c_{1}, c_{2}, \ldots, c_{n}$ be a centralising set of generators of $I$. If $n=1$ then the result follows by Corollary 1.3. If $n>1$ let $\bar{R}$ denote the ring $R /\left(c_{1} R\right), \bar{I}$ the ideal $I /\left(c_{1} R\right)$ and $Y$ the submodule ann $n_{X}\left(c_{1}\right)$. Clearly $Y$ is an $\bar{I}$-torsion right $\bar{R}$-module such that $\operatorname{ann}_{Y}(\bar{I})$ is Artinian. By induction on $n, Y$ is Artinian and by Corollary $1.3 X$ is Artinian.

Let $R$ be a ring and $I$ an ideal of $R$ with a centralising set of generators. By $(9,2.7)$ $I$ has the $A R$ property; that is, for every submodule $Y$ of a Noetherian right $R$-module $X$ there exists a positive integer $n$ such that $Y \cap X I^{n} \subseteq Y I$. It can easily be checked that if the ring $R$ is right Noetherian then a right $R$-module $X$ is $I$-torsion if and only if $\operatorname{ann}_{X}(I)$ is an essential submodule of $X$. If $R$ is any ring and $X$ is a monolithic right $R$-module with $U=\mu(X)$ then we shall denote the primitive ideal $\operatorname{ann}_{R}(U)$ by $\pi(X)$. With these remarks and notation we see that Lemma $1.4 \mathrm{im}$ mediately gives the following result.

Theorem 1.5. Let $R$ be a right Noetherian ring and $I$ be an ideal of $R$ such that $I$ has a centralising set of generators. Let $X$ be a monolithic right $R$-module such that $I \subseteq \pi(X)$ and $\operatorname{ann}_{X}(I)$ is Artinian. Then $X$ is Artinian.

Let $R$ be a right Noetherian ring and $X$ be a monolithic right $R$-module such that $P$ is the primitive ideal $\pi(X)$. If the ring $R / P$ is Artinian then $\operatorname{ann}_{X}(P)=\mu(X)$ since $\operatorname{ann}_{X}(P)$ is semisimple and monolithic in this case. Thus $\operatorname{ann}_{X}(P)$ is Artinian if $R / P$ is Artinian. For example, if $R$ is a commutative Noetherian ring then Theorem 1.5 reduces to the theorem of Matlis (8, Proposition 3 ) which states that finitely embedded $\boldsymbol{R}$-modules are Artinian and the proof is more elementary than that given by Matlis.

Now let $K$ be a field and $G$ be a finitely generated nilpotent group. If $R$ is the group ring $K G$ then $R$ is polycentral by (12, Theorem $\mathrm{A})$. Let $X$ be a monolithic right $R$-module. If $P=\pi(X)$ then $P$ is a maximal ideal of $R$ by (16, Theorem 3 ) and $\operatorname{ann}_{X}(P)$ is a monolithic right $R / P$-module. 


\section{Proof of Theorem $A$}

Let $R$ be a ring and $\mathfrak{X}$ a class of right $R$-modules. A right $R$-module $X$ is called a poly- $\mathfrak{X}$ module if and only if there exists a chain

$$
X=X_{0} \supseteq X_{1} \supseteq \cdots \supseteq X_{n-1} \supseteq X_{n}=0
$$

of submodules $X_{i}$ of $X$ such that the factor module $X_{i-1} / X_{i}$ lies in $\mathfrak{X}$ for each $1 \leqslant i \leqslant n$.

Lemma 2.1. Let $J$ be a ring and $H$ a normal subgroup of finite index in a group $G$. Let $X$ be a monolithic right JG-module. Then considered as a JH-module $X$ can be embedded in a finite direct sum of poly-monolithic right $\mathrm{JH}$-modules.

Proof. Let $R$ be the group ring $J G$ and $S$ the group ring $J H$. Let $U$ be the simple $R$-submodule of $X$. Suppose that $G=\cup_{i=1}^{t} x_{i} H$ for some positive integer $t$ and elements $x_{i}, 1 \leqslant i \leqslant t$, of $G$. If $u$ is a non-zero element of $U$ then $U=u R=\sum_{i=1}^{i} u x_{i} S$ and hence $U$ is a finitely generated right $S$-module. Let $V$ be a maximal $S$-submodule of $U$. Then $\cap_{i=1}^{i} V x_{i}$ is an $R$-submodule of $U$ and hence $\cap_{i=1}^{i} V x_{i}=0$. This gives a natural $S$-monomorphism $U \rightarrow \oplus_{i=1}^{i}\left(U / V x_{i}\right)$.

Thus there exist a positive integer $n$ and simple right $S$-submodules $U_{i}, 1 \leqslant i \leqslant n$, of $U$ such that $U=\oplus_{i=1}^{n} U_{i}$. Let us now define a chain $X=X_{0} \supseteq X_{1} \supseteq \cdots \supseteq X_{n-1} \supseteq X_{n}$ of $S$-submodules $X_{i}$ of $X$ by demanding that $X_{i}$ be an $S$-submodule of $X_{i-1}$ maximal with respect to the conditions $\oplus_{i=i+1}^{n} U_{j} \subseteq X_{i}$ and $U_{i} \cap X_{i}=0,1 \leqslant i \leqslant n$. Clearly for each $1 \leqslant i \leqslant n$ the right $S$-module $X_{i-1} / X_{i}$ is monolithic with $\mu\left(X_{i-1} / X_{i}\right) \cong U_{i}$. Moreover $X_{n} \cap U=0$. It follows that if $Y=\cap_{i=1}^{i} X_{n} x_{i}$ then $Y$ is an $R$-submodule of $X$ with $Y \cap U=0$ and hence $Y=0$. For each $1 \leqslant i \leqslant t, X / X_{n} x_{i}$ is a poly-monolithic right $S$-module. In addition there is a natural $S$-monomorphism $X \rightarrow \oplus_{i=1}^{t}\left(X / X_{n} x_{i}\right)$. This completes the proof.

Corollary 2.2. Let $J$ be a commutative Noetherian ring and $G$ be a finitely generated Abelian-by-finite group. Then every finitely embedded JG-module is Artinian.

Proof. The group $G$ has a finitely generated Abelian normal subgroup $A$ of finite index. The group ring $J A$ is a commutative Noetherian ring and by ( 8 , Proposition 3 ) (or Theorem 1.5 ) every finitely embedded $J A$-module is Artinian. The result follows by Lemma 2.1 .

Let $J$ be a ring and $H$ be a normal subgroup of a group $G$. We shall denote by $\omega H$ the right ideal of the group ring $J G$ generated by the elements $1-h$ with $h$ in $H$. It can easily be checked that $\omega H$ is the kernel of the canonical homomorphism of the group ring $J G$ onto the group ring $J(G / H)$, and thus $\omega H$ is a two-sided ideal of $J G$.

Proof of Theorem A. Without loss of generality we can suppose that $X$ is a monolithic right $J G$-module and, by Lemma 2.1 , that $G$ is finitely generated and nilpotent. Let $P=\pi(X)$ and $Q=P \cap J$. By (11, Corollary C3) $Q$ is a maximal ideal of 
$J$. If $R$ is the group ring $J G$ then $R$ is right Noetherian by (4, Theorem 1$)$ and the ideal $Q R$ of $R$ is generated by central elements of $R$. By Theorem 1.5 we can suppose without loss of generality that $Q=0$. Thus it is sufficient to prove the result when $J$ is a field.

Let $H$ be the normal subgroup $G \cap(1+P)$ of $G$. A theorem of Mal'cev (7, Theorem 1) shows that $G / H$ is Abelian-by-finite. Since $\operatorname{ann}_{x}(\omega H)$ is a monolithic module over the ring $J G / \omega H$ and since $J G / \omega H$ is isomorphic to the group ring $J(G / H)$ it follows by Corollary 2.2 that $\operatorname{ann}_{X}(\omega H)$ is Artinian. Clearly $\operatorname{ann}_{X}(P) \subseteq \operatorname{ann}_{X}(\omega H)$ and hence $\operatorname{ann}_{X}(P)$ is Artinian. By (12, Theorem A) $P$ has a centralising set of generators. Finally by Theorem $1.5 X$ is Artinian.

\section{Primitive images of group rings}

Let $K$ be a field and $G$ be a group. We are concerned in this section with primitive images of the group ring $K G$. Let us begin by observing that we can easily handle the situation when $K$ is a large field. Let $K$ be an algebraically closed field such that the cardinality of the set $K$ is greater than that of the set $G$. Let $P$ be a primitive ideal of $K G$. If the ring $K G / P$ is Artinian then there is a $K$-division algebra $D$ and a positive integer $n$ such that $K G / P \cong D_{n}$, the complete ring of $n \times n$ matrices with entries in $D$. Now $\operatorname{dim}_{K} D \leqslant \operatorname{dim}_{K} D_{n} \leqslant \operatorname{dim}_{K} K G=|G|<|K|$. Since $K$ is algebraically closed the proof of (10, Theorem 3) shows that $D=K$. Thus $\operatorname{dim}_{K}(K G / P)$ is finite. Thus, if $G$ is polycyclic and every primitive homomorphic image of $K G$ is Artinian then by (5, Theorem 3.3) $G$ is Abelian-by-finite.

We next extend a result of Zalesskii (16, Theorem 3) which states that if $K$ is a field and $G$ a finitely generated nilpotent group then every primitive ideal of $K G$ is a maximal ideal. We need the following lemma.

Lemma 3.1. Let $J$ be a right Noetherian ring and $H$ be a normal subgroup of finite index in a polycyclic-by-finite group $G$. Let $P \supset Q$ be prime ideals of JG. Then $P \cap J H \supset Q \cap J H$.

Proof. By (4, Theorem 1) $J G$ is a right Noetherian ring and hence $J G / Q$ is a prime right Noetherian ring. By $(3$, Theorem 10$)$ the ideal $P / Q$ contains a regular element $c+Q$ of $J G / Q$. Since $J G$ is a finitely generated right $J H$-module and $J H$ is a right Noetherian ring there exists a least positive integer $k$ such that

$$
S+c S+\cdots+c^{k} S+Q=S+c S+\cdots+c^{k} S+c^{k+1} S+Q,
$$

where $S$ is the group ring $J H$. It follows that there exist elements $a_{i}, 0 \leqslant i \leqslant k$, of $S$ such that $a_{0}+c a_{1}+\cdots+c^{k} a_{k}+c^{k+1}$ belongs to $Q$. If $a_{0} \in Q$ then $c\left(a_{1}+\cdots+c^{k-1} a_{k}+\right.$ $\left.c^{k}\right) \in Q$ and hence $a_{1}+\cdots+c^{k-1} a_{k}+c^{k} \in Q$, which contradicts the choice of $k$. It follows that $a_{0} \notin Q$. However $a_{0} \in P$ because $c \in P$ and we conclude that $P \cap S \supset$ $Q \cap S$.

Theorem 3.2. Let $J$ be a commutative Hilbert ring and $G$ a finitely generated nilpotent-by-finite group. Then every primitive ideal of JG is a maximal ideal. 
Proof. By (11, Corollary C3) we can suppose without loss of generality that $J$ is a field which we shall denote by $K$. Let $P$ be a primitive ideal of $K G$ and $X$ a simple right $K G$-module such that $P=\operatorname{ann}_{K G}(X)$. If $H$ is a normal nilpotent subgroup of finite index in $G$ then $H$ is finitely generated. Let $|G: H|=n$ and $T=$ $\left\{t_{1}=1, t_{2}, \ldots, t_{n}\right\}$ a transversal to the cosets of $H$ in $G$. By the proof of Lemma 2.1 there exists a maximal right $K H$-submodule $Y$ of $X$ such that $\cap_{i=1}^{n} Y t_{i}=0$ and a KH-monomorphism $X \rightarrow \oplus_{i=1}^{n}\left(X / Y t_{i}\right)$. If $Q=\operatorname{ann}_{K H}(X / Y)$ then $P \cap K H \subset Q$ and $\cap_{i=1}^{n} Q_{i}^{t_{i}} \subseteq P \cap K H$. Thus $P \cap K H=\cap_{i=1}^{n} Q^{i_{i}}$ where of course $Q$ is a primitive ideal of $K H$. By (16, Theorem 3) $Q$ is a maximal ideal of $K H$. Now let $M$ be a maximal ideal of $K G$ such that $P \subseteq M$. Then $\cap_{i=1}^{n} Q^{t_{i}} \subseteq M \cap K H$ and hence $Q^{\prime} \subseteq M \cap K H$ for some $t$ in $T$. Since $Q^{t}$ is a maximal ideal it follows that $Q^{t}=M \cap K H$ and hence $P \cap K H=M \cap K H$. By Lemma 3.1 we obtain the desired conclusion $P=M$.

Lemma 3.3. Let $J$ be $a$ ring and $G$ be a group such that every primitive homomorphic image of $J G$ is Artinian. Let $H$ be a normal subgroup of finite index in G. Then every primitive image of $\mathrm{JH}$ is Artinian.

Proof. Let $S$ denote the ring $J H$ and $R$ the ring $J G$. Let $P$ be a primitive ideal of $S$ and $M$ a maximal right ideal of $S$ such that $P=\operatorname{ann}_{S}(S / M)$. Since $M R$ is a proper ideal of $R$ it follows that there exists a maximal right ideal $M_{1}$ of $R$ such that $M R \subseteq M_{1}$ and hence $M=M_{1} \cap S$. If $Q=\operatorname{ann}_{R}\left(R / M_{1}\right)$ then $Q$ is a primitive ideal of $R$ and by hypothesis the ring $R / Q$ is Artinian. Moreover $Q \cap S \subseteq P$. There exists a positive integer $n$ and elements $x_{i}, 1 \leqslant i \leqslant n$, of $G$ such that $T=\left\{x_{1}, x_{2}, \ldots, x_{n}\right\}$ is a transversal to the cosets of $H$ in $G$. Let $\bar{S}=(S+Q) / Q$ and $\bar{x}_{i}=x_{i}+Q, 1 \leqslant i \leqslant n$. Then clearly $\bar{x}_{i} \bar{S}=\bar{S} \bar{x}_{i}, 1 \leqslant i \leqslant n$, and $R / Q=\bar{x}_{1} \bar{S}+\bar{x}_{2} \bar{S}+\cdots+\bar{x}_{n} \bar{S}$. By (2, Theorem 4) $\bar{S}$ is Artinian and hence $S /(Q \cap S)$ is Artinian. We conclude that $S / P$ is Artinian, as required.

Let $S$ be a simple ring and $\alpha$ an automorphism of $S$. Let $X$ be an indeterminate and let $R=S\left[X, X^{-1}, \alpha\right]$ denote the ring of polynomials $\Sigma_{i=s}^{i} \sigma_{i} X^{i}$ where $s \leqslant t$ are integers and $\sigma_{i} \in S, s \leqslant i \leqslant t$, multiplication being given by $X \sigma=\sigma^{\alpha} X, \sigma \in S$. We shall require the following fact about $R$.

Lemma 3.4. If the above ring $R$ contains a proper ideal $M$ such that $R / M$ is Artinian then $S$ is Artinian.

Proof. Since $R / M$ is Artinian it is clear that $M \neq 0$. Let $M_{1}=$ $S \cap\left(M+S X+S X^{2}+\cdots\right)$. Then $M_{1}$ is a non-zero ideal of $S$ and hence $M_{1}$ contains the identity 1 of $S$. That is, there exist elements $\sigma_{i}$ of $S$ such that $1+\sum_{i=1}^{m} \sigma_{i} X^{i} \in M$ and we infer that $X^{-m}+\sum_{i=1}^{m} \sigma_{i} X^{i-m} \in M$. Similarly there exist elements $\sigma_{i}^{\prime}$ of $S$ such that $X^{n}+\sum_{i=1}^{n} \sigma_{i}^{\prime} X^{n-i} \in M$. Since $M \cap S=0$ it follows by (2, Theorem 4) (see the proof of Lemma 3.3) that $S$ is Artinian.

If $H$ is a normal subgroup of a group $G$ and $J$ is a ring then an ideal $I$ of the group ring $J H$ is called $G$-invariant if and only if $I^{x}=x^{-1} I x \subseteq I$ for all $x$ in $G$. 
Corollary 3.5. Let $J$ be a ring and $G$ be a group such that every capital of $J G$ is Artinian. Let $H$ be a normal subgroup of $G$ such that $G / H$ is infinite cyclic and let $P$ be a $G$-invariant maximal ideal of $J H$. Then the ring $J H / P$ is Artinian.

Proof. Let $S$ be the ring $J H$ and $R$ the ring $J G$. Then $P R$ is a proper ideal of $R$ and hence $P R \subseteq M$ for some maximal ideal $M$ of $R$. It follows that $P=M \cap S$. Let $G / H$ be generated by the coset $x H$ and let $\alpha$ be the automorphism of $\bar{S}=S / P$ induced by conjugation by $x$. Then clearly $R / P R \cong \bar{S}\left[X, X^{-1}, \alpha\right]$. By Lemma 3.4, $S$ is Artinian.

Lemma 3.6. Let $K$ be a field and $G$ be a finitely generated nilpotent-by-finite group such that every capital of $K G$ is Artinian. Let $H$ be a normal subgroup of $G$ such that $G / H$ is infinite cyclic. Then every capital of $\mathrm{KH}$ is Artinian.

Proof. Let $S$ be the ring $K H$ and $R$ the ring $K G$. Let $P$ be a maximal ideal of $S$ and $N=\left\{x \in G: P^{x}=P\right\}$. Then $N$ is a normal subgroup of $G$ and $H \subseteq N$. If $H \neq N$ then $N$ has finite index in $G$ and by Theorem 3.2 and Lemma 3.3 every capital of $K N$ is Artinian. Corollary 3.5 applied to the group $N$ gives $S / P$ is Artinian. Now suppose that $H=N$. Let $M$ be a maximal right ideal of $S$ such that $P=\operatorname{ann}_{S}(S / M)$ and $M_{1}$ a maximal right ideal of $R$ such that $M=M_{1} \cap S$ (see the proof of Lemma 3.3). Let $A=R / M_{1}$ and $B=\left(S+M_{1}\right) / M_{1}$. If $Q=\operatorname{ann}_{R}(A)$ then $Q$ is a maximal ideal of $R$ by Theorem 3.2 and the ring $R / Q$ is Artinian by hypothesis.

Let $E_{1} \supseteq E_{2} \supseteq E_{3} \cdots$ be a descending chain of right ideals of $S$ such that each right ideal $E_{i}$ contains $P$. Then $E_{1} R \supseteq E_{2} R \supseteq E_{3} R \supseteq \cdots$ is a descending chain of right ideals of $R$ and there exists a positive integer $n$ such that $E_{n} R+Q=E_{n+1} R+Q=$ $E_{n+2} R+Q=\cdots$. Let $e \in E_{n}$. If $G / H$ is generated by the coset $H x$ then it is clear that $E_{n+1} R=\sum_{i=-\infty}^{\infty} E_{n+1} x^{i}$ and hence there exist integers $s \leqslant t$ and elements $f_{i}, s \leqslant i \leqslant t$, of $E_{n+1}$ and $q$ of $Q$ such that $e=\Sigma_{i=s}^{i} f_{i} x^{i}+q$. Then $b e=\Sigma_{i=s}^{i} b f_{i} x^{i}$ for each element $b$ of $B$. But by (11, Lemma 3) $A=\oplus_{i=-\infty}^{\infty} B x^{i}$ and it follows that $b\left(e-f_{0}\right)=0$ for each element $b$ of $B$. Hence $e-f_{0} \in P$ and $e \in E_{n+1}$. It follows that $E_{n}=E_{n+1}=E_{n+2}=\ldots$ and the ring $S / P$ is Artinian, as required.

Lemma 3.7. Let $K$ be a field and $G$ be a polycyclic-by-finite group such that for every subnormal subgroup $H$ of $G$ every primitive homomorphic image of $K H$ is Artinian. Then either $K$ is absolute or $G$ is Abelian-by-finite.

Proof. Suppose $K$ is not absolute and $G$ is not Abelian-by-finite. By adapting Hall's proof of (5, Theorem 3.3) we can suppose without loss of generality that $G$ is generated by a free Abelian normal subgroup $A$ and an element $z$. Let $X$ be Hall's simple right $K G$-module with basis $\left\{v_{m}: m \in Z\right\}$ (see (5, p. 616)), and note that $v_{m} z=v_{m+1}$ for all $m$. If $R=K G$ and $P=\operatorname{ann}_{R}(X)$ then $P$ is a primitive ideal of $R$ and we claim that the ring $R / P$ is not Artinian. Consider the chain of left ideals $R(1-z)+P \supseteq R\left(1-z^{2}\right)+P \supseteq R\left(1-z^{4}\right)+P \supseteq \cdots$ and suppose that $n$ is a positive integer such that $R\left(1-z^{n}\right)+P=R\left(1-z^{2 n}\right)+P$. Then $X\left(1-z^{n}\right)=X\left(1-z^{2 n}\right)$. In particular

$$
v_{0}\left(1-z^{n}\right)=\left(\sum_{i=s}^{1} k_{i} v_{i}\right)\left(1-z^{2 n}\right)
$$


for some integers $s \leqslant t$ and elements $k_{i}, s \leqslant i \leqslant t$, of $K$ with $k_{s}, k_{t} \neq 0$. That is,

$$
v_{0}-v_{n}=\sum_{i=s}^{l} k_{i} v_{i}-\sum_{i=s}^{t} k_{i} v_{i+2 n}
$$

which is impossible. Thus $R / P$ is not Artinian, as required.

Proof of Theorem B. Let $K$ be a field and $G$ be a finitely generated nilpotent-byfinite group such that every capital of $K G$ is Artinian. If $H$ is a subnormal sub-group of $G$ then there is a chain $H=H_{0} \triangleleft H_{1} \triangleleft \cdots \triangleleft H_{n}=G$ such that each factor $H_{i} / H_{i-1}$, $1 \leqslant i \leqslant n$, is either finite or cyclic. By Theorem 3.2 and Lemmas 3.3 and 3.6 every capital of $\mathrm{KH}$ is Artinian. Theorem 3.2 and Lemma 3.7 combine to complete the proof.

\section{Proof of Theorem $\mathrm{C}$}

It is a well known fact that if $c$ is a regular element of a ring $R$ and $X$ is an injective right $R$-module then $X=X c$.

Suppose that $J$ is a CHACA ring and $G$ is a polycyclic-by-finite group such that there exists a non-zero finitely generated injective right $J G$-module $X$. Our aim is to prove that $\operatorname{ann}_{J}(X)$ contains a finite product of maximal ideals and $G$ is finite. It is well known that because $J G$ is a right Noetherian ring $X$ is a finite direct sum of indecomposable injective right $J G$-modules. Thus without loss of generality we can suppose that $X$ is indecomposable.

Firstly we prove that $X$ is Artinian. If $P$ is maximal in $\operatorname{ann}_{J G}(Y): Y$ is a non-zero submodule of $X$ \} then $P$ is a prime ideal of $J G$. Suppose that $P$ is not a primitive ideal and let $S$ be the prime right Noetherian ring $J G / P$. If $A=\operatorname{ann}_{x}(P)$ then $A$ is a finitely generated injective right $S$-module. If $a$ is a non-zero element of $A$ then there exists a maximal right ideal $M$ of $S$ such that $\operatorname{ann}_{S}(a) \subseteq M$. Let $Q$ be the primitive ideal $\operatorname{ann}_{S}(S / M)$ of $S$. By $\left(6\right.$, Theorem $\left.6^{\prime}\right)$ there exists an ideal $I$ of $S$ such that $I \subseteq Q, I$ has the $A R$ property and the ring $S / I$ is right Artinian. Since $I$ is a non-zero ideal of $S$ it follows that $I$ contains a regular element by $(3$, Theorem 10$)$. Thus by the above remark $A=A I$. There exists a positive integer $n$ such that $a R \cap A I^{n} \subseteq a I$ and hence $a(1-i)=0$ for some element $i$ of $I$, and this contradicts $I \subseteq M$. Thus $P$ is a primitive ideal of $J G$. By (11, Corollary $A$ ) $J G / P$ is an Artinian ring and it follows that $X$ has non-zero socle. Therefore $X$ is monolithic and by $\left(6\right.$, Theorem $\left.6^{\prime}\right) X$ is Artinian.

Clearly $P=\pi(X)$ and by (11, Corollary C3) $P \cap J$ is a maximal ideal of $J$. By (9, 2.7) there exists a positive integer $m$ such that $(P \cap J)^{m} \subseteq$ ann $_{J}(X)$. Moreover, $X$ has a composition series and by $(11$, Corollary $A)$ the set $H$ of all elements $g$ of $G$ such that $X(1-g)=0$ is a normal subgroup of finite index in $G$. If an element $h$ of $H$ has infinite order then $1-h$ is a regular element of $J G$ and hence $X=X(1-h)=0$. Thus $H$ is periodic and hence finite. Therefore $G$ is finite and this completes the proof of Theorem C.

\section{Added in proof:}

Corollary A was proved independently for the case when $J$ is the ring of integers by $R$. L. Snider (Injective hulls of simple modules over group rings, in Ring Theory edited by S. K. Jain and K. E. Eldridge (Dekker, 1977), pp. 223-226). 


\section{REFERENCES}

(1) I. G. CONNELL, On the group ring, Canad. J. Math. 15 (1963), 650-685.

(2) E. Formanek and A. V. JATEgaonkar, Subrings of Noetherian rings, Proc. Amer. Math. Soc. 46 (1974), 181-186.

(3) A. W. GoldIE, The structure of prime rings under ascending chain conditions, Proc. London Math. Soc. (3)8 (1958), 589-608.

(4) P. Hall, Finiteness conditions for soluble groups, Proc. London Math. Soc. (3)4 (1954), 419-436.

(5) P. Hall, On the finiteness of certain soluble groups, Proc. London Math. Soc. (3)9 (1959), 595-622.

(6) A. V. JATEGAONKAR, Integral group rings of polycyclic-by-finite groups, J. Pure Appl. Algebra 4 (1974), 337-343.

(7) A. I. MAL'CEV, On certain classes of infinite soluble groups, Mat. Sb. 28 (1951), 567-588 (Amer. Math. Soc. Translations (2)2 (1956), 1-21).

(8) E. MATLIS, Modules with descending chain condition, Trans. Amer. Math. Soc. 97 (1960), 495-508.

(9) Y. NOUAZE and P. GABRIEL, Ideaux premiers de l'algèbre enveloppante d'une algèbre de Lie nilpotente, J. Algebra 6 (1967), 77-99.

(10) D. S. Passman, Primitive group rings, Pacific J. Math. 47 (1973), 499-506.

(11) J. E. Roseblade, Group rings of polycyclic groups, J. Pure Appl. Algebra 3 (1973), 307-328.

(12) J. E. Roseblade and P. F. Smith, A note on hypercentral group rings, J. London Math. Soc. (2)13 (1976), 183-190.

(13) A. RosEnBERG and D. ZELINSKY, Finiteness of the injective hull, Math. Z. 70 (1959), 372-380.

(14) R. L. SNIDER, Primitive ideals in group rings of polycyclic groups, Proc. Amer. Math. Soc. 57 (1976), 8-10.

(15) P. VÁmos, The dual of the notion of 'finitely generated', J. London Math. Soc. 43 (1968), 643-646.

(16) A. E. ZALESSKII, Irreducible representations of finitely generated nilpotent torsionfree groups, Math. Notes 9 (1971), 117-123.

UNIVERSITY OF GLASGOW

Glasgow G12 8QW 